\title{
Evaluación de la seguridad de la CPRE en pacientes de la tercera edad: experiencia de un hospital universitario en Bogotá
}

\section{Evaluation of the Safety of ERCP in Older Patients in the Experience of a University Hospital in Bogota}

Viviana Parra, MD, ${ }^{1}$ Margarita Huertas Q., MD, ${ }^{2}$ Johnny Beltrán, MD, ${ }^{3}$ Douglas Ortiz, MD. ${ }^{2}$

1 Residente de Cirugía General, Universidad Militar Nueva Granada. Bogotá, Colombia. Correo electrónico: vivi 850@hotmail.com

2 Especialista en Cirugía General-Gastroenterología, Departamento de Cirugía General Gastroenterología, Hospital Universitario Clínica San Rafael. Bogotá, Colombia.

${ }^{3}$ Especialista en Medicina Interna-Epidemiología.

${ }^{4}$ Especialista en Cirugía General-Gastroenterología. Jefe, Departamento de Gastroenterología, Hospital Universitario Clínica San Rafael. Bogotá, Colombia.

Fecha recibido: $\quad 05-11-14$

Fecha aceptado: 06-04-15

\section{Resumen}

Antecedentes: la colangiopancreatografía retrógrada endoscópica (CPRE) es el método de elección en el manejo de la coledocolitiasis; sin embargo, existen controversias sobre su seguridad en el adulto mayor.

Objetivo: determinar si hay mayor riesgo de complicaciones con la realización de la CPRE en el manejo de la coledocolitiasis en pacientes mayores de 70 años de edad valorados en el Hospital Universitario Clínica San Rafael (HUCSR).

Metodología: se realizó un estudio analítico de corte transversal que incluyó pacientes que ingresaron al servicio de urgencias del HUCSR durante el período de 1 año (2011-2012) con diagnóstico de coledocolitiasis. La información demográfica, clínica y paraclínica, así como los hallazgos del procedimiento, fueron analizados teniendo en cuenta dos grupos de pacientes (mayores y menores de 70 años de edad). Se llevó a cabo un análisis estadístico univariado y bivariado.

Resultados: se incluyó un total de 171 pacientes. La presencia de comorbilidades (es decir, diabetes e hipertensión arterial), los niveles de bilirrubina directa y de transaminasas fueron variables asociadas significativamente con los pacientes con edad mayor o igual a los 70 años. Se presentaron complicaciones relacionadas con el procedimiento, siendo la más frecuente la pancreatitis pos-CPRE y la hemorragia pospapilotomía; sin embargo, no estuvieron relacionadas con la edad de los pacientes.

Conclusión: la CPRE es segura en pacientes con coledocolitiasis. La edad mayor de 70 años no incrementa la mortalidad relacionada con el procedimiento.

\section{Palabras clave}

Colangiopancreatografía retrógrada endoscópica (CPRE), coledocolitiasis, tercera edad, complicaciones.

\section{Abstract}

Background: Although endoscopic retrograde cholangiopancreatography (ERCP) is the method of choice for management of choledocholithiasis, there are still controversies about its safety for elderly patients.

Objective: To determine whether there is increased risk of complications from ERCP in the management of choledocholithiasis in patients over 70 years valued at University Hospital Clínica San Rafael (HUCSR).

Methodology: A cross-sectional analytical study that included patients admitted to the emergency department of HUCSR during the period of one year (2011-2012) with a diagnosis of choledocholithiasis was performed. The demographic, clinical and paraclinical findings and procedure were analyzed taking into account two groups of patients (older and younger than 70 years). Univariate statistical analysis was performed and bivariate.

Results: A total of 171 patients were included. The presence of comorbidities (eg, diabetes and hypertension), the levels of direct bilirubin and transaminases were variables significantly associated with patients with greater than or equal to 70 years old. Procedure-related complications were presented the most frequent post-ERCP pancreatitis and post papilotomy bleeding; however, were not related to patient age.

Conclusion: ERCP is safe in patients with choledocholithiasis. Age over 70 years does not increase the mortality associated with the procedure.

\section{Keywords}

Endoscopic retrograde cholangiopancreatography (ERCP), choledocholithiasis, elderly, complications. 


\section{INTRODUCCIÓN}

La patología biliar es un motivo de consulta frecuente en pacientes de la tercera edad, dado que existen cambios en su anatomía y fisiología biliar que crean mayor predisposición para el desarrollo de algunas enfermedades como la coledocolitiasis (1). Estos cambios incluyen algunos, a saber: mayor litogenicidad, disminución en la síntesis de sales biliares, alteración en la motilidad de la vesícula biliar, presencia de divertículos peripapilares, disminución en la respuesta a la colecistoquinina y aumento en el diámetro del colédoco.

La colangiopancreatografía retrógrada endoscópica (CPRE) se ha establecido como el método de elección para el tratamiento de coledocolitiasis mostrando una tasa de extracción exitosa entre el $85 \%$ y el $90 \%$ de los casos (2). Sin embargo, como todo procedimiento invasivo, tiene complicaciones, las cuales han sido reportadas en el rango del 5\% al $10 \%$, por lo que la CPRE se reserva a los pacientes con coledocolitiasis de alta probabilidad o confirmada (3). En cuanto a las complicaciones más frecuente se encuentran la pancreatitis pos-CPRE (5\%) (3), la hemorragia posesfinterotomía (2\%) (4) y la perforación (1\%) (3). Otras complicaciones asociadas con el procedimiento como la colangitis y la colecistitis pos-CPRE, los eventos cardiopulmonares, los efectos secundarios a la sedación y muerte, han sido reportadas con una frecuencia menor $(0,5 \%)(3)$.

Los estudios que tratan de valorar la eficacia, seguridad y tasa de complicaciones de la CPRE en pacientes mayores de 70 años de edad son escasos, con un tamaño muestral inapropiado y, hasta el momento, no han sido valorados en una población con alta mezcla étnica, como la nuestra. Por tanto, no existe un consenso sobre los factores asociados (por ejemplo, comorbilidades) con la presentación de dichas complicaciones. El objetivo del presente estudio fue establecer cuáles son estos factores asociados y su frecuencia en pacientes mayores de 70 años de edad sometidos a CPRE, con el fin de ofrecer un perfil de mayor seguridad y generar propuestas de acción para disminuir las tasas de complicación.

\section{MATERIALES Y MÉTODOS}

\section{Población del estudio}

Este fue un estudio analítico de tipo corte transversal, en el cual 171 pacientes fueron incluidos de manera consecutiva durante el período comprendido entre septiembre de 2011 y agosto de 2012 en el Hospital Universitario Clínica San Rafael (HUCSR). Todos los pacientes fueron pacientes adultos ingresados por urgencias, que cumplieron al menos una de las siguientes condiciones (5): obstrucción biliar y/o colangitis aguda, pancreatitis de origen biliar, coledocolitiasis diagnosticada o con sospecha elevada por ecogra- fía, tomografía axial computarizada (TAC), ecoendoscopia y/o colangiorresonancia o diagnóstico clínico de coledocolitiasis sintomática (es decir, cólico biliar + colelitiasis + dilatación ecográfica de la vía biliar $>7 \mathrm{~mm}+$ alteración del perfil hepático + presencia de ictericia, acolia y/o coluria).

El diseño del estudio fue aprobado por el Comité Institucional en Investigación del HUCSR.

Cada paciente fue valorado por 2 endoscopistas con experiencia. La información sociodemográfica, clínica y paraclínica fue obtenida por entrevista, revisión de la historia clínica, examen físico, además de la derivada propia de los hallazgos del procedimiento. Todos los datos fueron colectados en una base de datos electrónica.

\section{Procedimiento: CPRE}

El procedimiento fue realizado por 2 endoscopistas con experiencia en CPRE y certificación en sedación. A todos los pacientes se les suministró lidocaína tópica bucofaríngea en espray al $10 \%$, sedación con midazolam (1-3 mg) o meperidina $(30-50 \mathrm{mg}$ ) según criterio del endoscopista, y dosis única de antiespasmódico con butilbromuro de hioscina (20 $\mathrm{mg}$ ). El procedimiento se realizó con estricta monitorización del paciente, según los protocolos de la institución.

El duodenoscopio utilizado fue Olympus Evis-Exera (Olympus Inc., Tokio, Japón). Luego de la canulación de la vía biliar se realizó la colangiografía para verificar la presencia de coledocolitiasis. En caso de ser confirmada, se llevó a cabo la extracción de los cálculos con canastilla de Dormia o balón de extracción.

Todos los pacientes continuaron hospitalizados durante un período promedio de 2 días, con un período de ayuno mínimo de 24 horas.

Se valoró el éxito o fracaso del procedimiento, la presencia de alteraciones anatómicas que dificultaron el procedimiento (es decir, divertículo periampular, fístula peripapilar, entre otros), la realización de papilotomía y la presencia o no de alguna de las complicaciones pos-CPRE.

\section{Complicaciones}

Se considera como complicación a todo evento adverso relacionado con la CPRE que ocurre durante los 30 días ulteriores a su realización (3). La mortalidad atribuible al procedimiento es definida como aquella que ocurre en los 30 días ulteriores a su realización o como resultado de una complicación propia del procedimiento (6). En cuanto a las complicaciones que fueron evaluadas en el estudio, se encuentran la pancreatitis pos-CPRE, considerada como la aparición de un nuevo dolor abdominal tipo pancreático o empeoramiento del dolor existente, asociado con al menos un incremento de tres veces la ami- 
lasa sérica o lipasa del valor de referencia en las primeras 24 horas después de la CPRE $(4,7)$; la perforación duodenal, que se puede comprobar durante el procedimiento o por manifestaciones clínicas y radiología compatible (es decir, dolor abdominal persistente con verificación de neumoperitoneo) (4). También se tuvo en cuenta la hemorragia posesfinterotomía, que puede ser temprana o diferida. La temprana se considera como un sangrado que se documenta durante el procedimiento, mientras que la diferida es la presencia de hemorragia digestiva (por ejemplo, melenas o hematemesis) después de las 24 horas del procedimiento $(4,7)$. La colangitis pos-CPRE es aquella que debe tener ausencia clínica o imagenológica de colangitis previa al procedimiento y que conlleve la realización urgente de alguna intervención $(4,8,9)$. Por su parte, la colecistitis aguda también debe tener ausencia clínica o imagenológica de colecistitis previa al procedimiento, que lleve a la realización urgente de colecistectomía o drenaje de la vesícula (4). Por último, también fueron evaluadas las complicaciones cardiopulmonares y las secundarias a la sedación. Estas complicaciones incluyen: infarto agudo de miocardio (IAM), evento cerebrovascular (ECV), insuficiencia respiratoria, arritmia, tromboembolismo pulmonar y broncoaspiración (10).

\section{Análisis estadístico}

En primer lugar, se llevó a cabo un análisis univariado. Las variables categóricas se analizaron mediante frecuencias. La prueba de Kolmogorov-Smirnov se realizó para evaluar la normalidad de las variables cuantitativas continuas. Los datos paramétricos se expresan con la media y la desviación estándar (DS), y los datos no paramétricos se describen como mediana y rango intercuartil (RIC).

En segundo lugar, se hicieron análisis bivariados para buscar asociaciones respecto a las complicaciones del procedimiento entre aquellos pacientes menores y mayores de 70 años de edad. La presencia de complicaciones y/o mortalidad se evaluó mediante pruebas de chi cuadrado o la prueba exacta de Fisher cuando la variable fue dicotómica. Los valores paramétricos se analizaron mediante la prueba $\mathrm{T}$ Student. Los valores no paramétricos fueron evaluados con la prueba U-Mann-Whitney. Un valor de $p<0,05$ fue considerado significativo.

Los análisis estadísticos se realizaron utilizando el paquete estadístico para las Ciencias Sociales (SPSS v.20, Chicago, IL).

\section{RESULTADOS}

\section{Descripción de los pacientes llevados a CPRE}

En total se llevaron a cabo 312 CPRE. Sin embargo, 141 pacientes fueron excluidos del análisis pues fueron manejados de forma ambulatoria $(\mathrm{n}=92)$, tuvieron otra indicación para la realización de CPRE $(\mathrm{n}=23)$ y no tenían datos completos en la historia clínica $(n=26)$. Por tanto, un total de 171 pacientes cumplieron con todos los criterios de selección del estudio.

Del total de la población, el $64,3 \%$ fueron mujeres $(110 / 171)$. Los pacientes con edad mayor o igual a 70 años corresponde a un $28 \%(48 / 171)$. Las comorbilidades más frecuentemente encontradas corresponden a hipertensión arterial y diabetes mellitus, 26,9\% (46/171) y 9,9\% (17/171), respectivamente. Para una descripción detallada véase la tabla 1.

Tabla 1. Características clínicas de los pacientes por edad que se sometieron a CPRE

\begin{tabular}{lccccccc}
\hline \multicolumn{1}{c}{ Variable } & $\mathbf{n .}^{\circ}$ & $\%$ & $\begin{array}{c}\mathrm{n} \cdot{ }^{\circ} \\
\text { (menores de } \mathbf{7 0} \text { años de edad) }\end{array}$ & $\%$ & $\begin{array}{c}\mathrm{n} \cdot{ }^{\circ} \\
\text { (mayores de } \mathbf{7 0} \text { años de edad) }\end{array}$ & $\begin{array}{c}\text { Valor } \\
\text { de } \mathbf{p}\end{array}$ \\
\hline Género masculino & 61 & $35,6 \%$ & 40 & $32,8 \%$ & 21 & $42,9 \%$ & 0,16 \\
Diabetes & 17 & $9,9 \%$ & 4 & $3,3 \%$ & 13 & $26,5 \%$ & 0 \\
HTA & 46 & $26,9 \%$ & 18 & $14,8 \%$ & 28 & $57,1 \%$ & 0 \\
Insuficiencia cardíaca & 3 & $1,7 \%$ & 1 & $0,8 \%$ & 2 & $4,1 \%$ & 0,19 \\
Cardiopatía isquémica & 2 & $1,1 \%$ & 1 & $0,8 \%$ & 1 & $2,0 \%$ & 0,48 \\
ECV & 2 & $1,1 \%$ & 0 & $0,0 \%$ & 2 & $4,1 \%$ & 0,07 \\
EPOC & 4 & $2,3 \%$ & 2 & $1,6 \%$ & 2 & $4,1 \%$ & 0,31 \\
Cáncer & 4 & $2,3 \%$ & 2 & $1,6 \%$ & 2 & $4,1 \%$ & 0,13 \\
ERC & 7 & $4,0 \%$ & 2 & $1,6 \%$ & 5 & $10,2 \%$ & 0,01 \\
Hepatopatía & 2 & $1,1 \%$ & 1 & $0,8 \%$ & 1 & $2,0 \%$ & 0,01 \\
ASA & 6 & $3,5 \%$ & 3 & $2,5 \%$ & 3 & $6,1 \%$ & 0 \\
Clopidogrel & 1 & $0,5 \%$ & 1 & $0,8 \%$ & 0 & $0 \%$ & 1 \\
\hline
\end{tabular}

ASA: ácido acetil salicílico; ECV: evento cerebrovascular; EPOC: enfermedad pulmonar obstructiva crónica; ERC: enfermedad renal crónica; HTA: hipertensión arterial. 


\section{Comparación entre grupos de edades (análisis bivariado)}

En cuanto a las comorbilidades, se encontró una frecuencia estadísticamente mayor de insuficiencia renal crónica y hepatopatía en pacientes mayores de 70 años de edad respecto al otro grupo $(10,2 \%$ versus $1,6 \%, p=0,01$ y $2,0 \%$ versus $\mathrm{p}=0,01$ y $0.8 \%$, respectivamente) (tabla 1 ).

Se analizó el perfil hepático de los pacientes a su ingreso a urgencias y se encontró que los valores de la bilirrubina directa y la transaminasa TGO fueron significativamente más altos en la población de mayores de 70 años de edad $(3,8$ versus $2,9, \mathrm{p}=0,05 ; 283$ versus $171, \mathrm{p}=0,01$, respectivamente). Para el caso de la transaminasa TGP, la asociación fue inversa aunque estadísticamente significativa (207 versus $382 ; \mathrm{p}<0,001)$. No se observaron diferencias en los valores de bilirrubina total, fosfatasa alcalina, amilasa y en el diámetro del colédoco entre los dos grupos.

Respecto a los hallazgos endoscópicos, aproximadamente un tercio de los pacientes requirió precorte en la papila, siendo más frecuente en los mayores de 70 años de edad $(38,8 \%$ versus $19,7 \% ; p=0,007)$. Adicionalmente se encontró una mayor presencia de divertículos peripapilares en pacientes del mismo grupo etario ( $16,3 \%$ versus $5,7 \%$; $p=0,034)$. En cuanto a la papilotomía, esfinteroplastia y fístula peripapilar, no se hallaron asociaciones estadísticamente significativas. Del total de procedimientos realizados, se logró una extracción completa en la primera CPRE en 128 pacientes $(74,8 \%)$; de estos, el $81,1 \%$ eran menores de 70 años y $18,9 \%$ mayores o iguales a 70 años de edad $(p=0,001)$. La CPRE fue fallida en el primer tiempo en $43(25,1 \%)$ casos. De este porcentaje, a 21 pacientes se les realizó un segundo tiempo de CPRE, la cual fue exitosa en 18 y fallida en 3 casos, sin mostrar diferencias importantes en las edades (tabla 2).

De las colangiografías llevadas a cabo, un $8,1 \%$ de los pacientes presentó complicaciones (14/171) y un $1,75 \%$ falleció (3/171). Las complicaciones más frecuentemente presentadas fueron la pancreatitis pos-CPRE y la hemorragia pospapilotomía (3,5\%, cada una). Respecto a

Tabla 2. Hallazgos en la CPRE por edad

\begin{tabular}{|c|c|c|c|c|c|c|c|}
\hline Hallazgo & n. ${ }^{\circ}$ total & $\%$ & $\begin{array}{c}\mathrm{n}^{\circ} \\
\text { (menores de } 70 \text { años de edad) }\end{array}$ & $\%$ & $\begin{array}{c}\mathrm{n} .{ }^{\circ} \\
\text { (mayores de } 70 \text { años de edad) }\end{array}$ & $\%$ & $\begin{array}{l}\text { Valor } \\
\text { de } p\end{array}$ \\
\hline Papilotomía & 161 & $94,1 \%$ & 117 & $95,9 \%$ & 44 & $89,8 \%$ & 0,469 \\
\hline Precorte & 43 & $25,1 \%$ & 24 & $19,7 \%$ & 19 & $38,8 \%$ & 0,007 \\
\hline Éxito de la CPRE 1 & 128 & $74,8 \%$ & 99 & $81,1 \%$ & 29 & $59,2 \%$ & 0,001 \\
\hline Éxito de la CPRE 2 & 18 & $10,5 \%$ & 10 & $8,2 \%$ & 8 & $16,3 \%$ & 1 \\
\hline Esfinteroplastia & 11 & $6,4 \%$ & 6 & $4,9 \%$ & 5 & $10,2 \%$ & 0,295 \\
\hline Fístula peripapilar & 4 & $2,3 \%$ & 3 & $2,5 \%$ & 1 & $2,0 \%$ & 1 \\
\hline Divertículo & 15 & $8,7 \%$ & 7 & $5,7 \%$ & 8 & $16,3 \%$ & 0,034 \\
\hline
\end{tabular}

CPRE: colangiopancreatografía retrógrada endoscópica.

Tabla 3. Complicaciones pos-CPRE

\begin{tabular}{lcccccc}
\hline & n. ${ }^{\circ}$ total & Mayores de $\mathbf{7 0}$ años de edad & $\%$ & Menores de $\mathbf{7 0}$ años de edad & $\%$ & Valor de $\mathbf{p}$ \\
\hline Complicación & 14 & 6 & $27 \%$ & 7 & $54 \%$ & 0,195 \\
Colangitis pos-CPRE & 1 & 1 & $100 \%$ & 0 & $0 \%$ & 0,281 \\
Pancreatitis pos-CPRE & 6 & 3 & $50 \%$ & 3 & $50 \%$ & 0,351 \\
Perforación duodenal & 1 & 0 & $0 \%$ & 1 & $100 \%$ & 1 \\
Hemorragia pospapilotomía & 6 & 2 & $33 \%$ & 4 & $67 \%$ & 0,674 \\
Colecistitis pos-CPRE & 0 & 0 & $0 \%$ & 0 & $0 \%$ & - \\
IAM & 0 & 1 & $100 \%$ & 0 & $0 \%$ & 0,281 \\
Arritmia & 0 & 2 & $100 \%$ & 0 & $0 \%$ & 0,078 \\
Embolismo aéreo & 0 & 0 & $0 \%$ & 0 & $0 \%$ & - \\
Broncoaspiración & 0 & 0 & $0 \%$ & 0 & $0 \%$ & - \\
TEP & 0 & 0 & $0 \%$ & 0 & $0 \%$ & - \\
Muerte & 3 & 2 & $67 \%$ & 1 & $33 \%$ & 0,191 \\
\hline
\end{tabular}

CPRE: colangiopancreatografía retrógrada endoscópica; IAM: infarto agudo de miocardio; TEP: tromboembolismo pulmonar. 
la pancreatitis, de acuerdo con el cálculo de APACHE II para severidad, 4 pacientes tuvieron pancreatitis leve y 2 , pancreatitis severa. Estos últimos 2 pacientes fallecieron como consecuencia de la pancreatitis $(1,6 \%)$. La identificación de hemorragia pospapilotomía en todos los casos fue temprana y oportuna, por lo que se logró un control adecuado de la misma mediante escleroterapia en el mismo procedimiento sin intervenciones adicionales. Todos estos pacientes fueron llevados a control endoscópico, donde se corroboró el control del sangrado en el $100 \%$ de los casos sin necesidad de terapia transfusional. Sin embargo, respecto a las complicaciones, ninguna presentó frecuencias significativas en los dos grupos etarios de estudio (tabla 3 ).

Los 3 pacientes fallecidos fueron el 100\% del género femenino. Como factores de riesgo hallados para mortalidad, independientemente de la edad, se encontró la pancreatitis pos-CPRE, las arritmias y el infarto agudo de miocardio, fueron las asociaciones significativamente encontradas (tabla 4).

Tabla 4. Factores de riesgo independientes para mortalidad

\begin{tabular}{lcccc}
\hline \multicolumn{1}{c}{ Variable } & Mortalidad & $\begin{array}{c}\text { n. }{ }^{\circ} \text { de } \\
\text { casos }\end{array}$ & $\begin{array}{c}\text { \% dentro de los } \\
\text { muertos }\end{array}$ & $\begin{array}{c}\text { Valor } \\
\text { de } \mathbf{p}\end{array}$ \\
\hline Sexo & Sí & 0 & $0,0 \%$ & 0,553 \\
masculino & No & 3 & $2,7 \%$ & \\
Mayor de 70 & Sí & 2 & $4,2 \%$ & 0,191 \\
años de edad & No & 1 & $0,8 \%$ & \\
Pancreatitis & Sí & 2 & $33,3 \%$ & 0,003 \\
pos-CPRE & No & 1 & $0,6 \%$ & \\
IAM & Sí & 1 & $100 \%$ & 0,018 \\
& No & 2 & $1,2 \%$ & \\
Arritmia & Sí & 1 & $50 \%$ & 0,035 \\
Hemorragia & No & 2 & $1,2 \%$ & \\
Perforación & Sí & 0 & $0,0 \%$ & 1 \\
& No & 3 & $1,8 \%$ & \\
& Sí & 0 & $0,0 \%$ & 1 \\
\hline
\end{tabular}

CPRE: colangiopancreatografía retrógrada endoscópica; IAM: infarto agudo de miocardio.

\section{DISCUSIÓN}

\section{Características clínicas de los pacientes}

Nuestro estudio identificó una mayor frecuencia de comorbilidades, así como un mayor consumo de ASA entre las personas mayores de 70 años de edad. Estos hallazgos son concordantes con la literatura publicada, en la cual se aprecia una mayor prevalencia de las mismas a una mayor edad de los pacientes llevados a CPRE $(11,12)$. Recientemente se identificaron factores pronósticos similares a los hallados en nuestra población, a saber: edad (incrementa el riesgo en $0,8 \%$ por año de vida), género masculino (odds ratio [OR] 1,2; IC 95\%, 1,1-1,2), diabetes (OR 1,1; IC 95\%, 1-1,2) e hipertensión arterial (OR 1,1; IC 95\%, 1-1,2) (13).

\section{Laboratorios al ingreso}

Los valores de fosfatasa alcalina, TGP, TGO y bilirrubina total son reconocidos como pruebas paraclínicas alteradas en los casos de coledocolitiasis (14).

Se encontró que en las personas mayores de 70 años de edad, los niveles de TGP, TGO y bilirrubina total son significativamente mayores. Los niveles de bilirrubina total, directa e indirecta, están directamente relacionados con un proceso oxidativo más fuerte en los pacientes con coledocolitiasis; es decir, los hallazgos del presente estudio sugieren que los pacientes mayores de 70 años de edad están expuestos a un mayor proceso de daño tisular y podrían tener niveles más altos de daño hepático (15).

\section{Hallazgos endoscópicos, hallazgos en CPRE y procedimiento}

En este estudio se identificó un mayor número de pacientes con divertículo peripapilar en mayores de 70 años de edad. Se ha reportado que la presencia de estos puede estar relacionada con una mayor probabilidad de coledocolitiasis recurrente (16). En un estudio previo, en el cual se clasificaron a los pacientes en 2 grupos; mayores y menores de 75 años de edad, no se observaron diferencias estadísticamente significativas en la presencia de estos. Dicha diferencia con los resultados de nuestro estudio podría ser explicada por las características propias de la población del estudio (por ejemplo, edad, severidad de la enfermedad y comorbilidades) o bien por la manera en la cual fueron clasificados los pacientes (17).

El presente trabajo mostró una extracción exitosa en un primer tiempo en el $75 \%$ de los pacientes y un aumento del $10 \%$ en la eficacia en el segundo tiempo. Se encontró además que el éxito en el primer tiempo es significativamente mayor en menores de 70 años de edad. Algunos estudios han explorado los factores relacionados con la falla en la extracción exitosa en el primer tiempo de realización de la CPRE. La esfinteroplastia, la presencia de divertículo peripapilar y el tamaño y número de los cálculos son factores que reducen el éxito en el primer tiempo de la CPRE, según publicaciones recientes (18). El mayor éxito reportado en pacientes menores de 70 años de edad puede estar relacionado con alguno de estos factores reportados en otros estudios.

El éxito reportado de la CPRE en el primer y segundo tiempo (85\%) es similar al reportado en la literatura (80\%$100 \%)(19-21)$. Aunque estos estudios reportan una menor 
frecuencia de necesidad de realización de un segundo tiempo de la CPRE, la diferencia puede estar relacionada con las características propias de la población incluida en el presente estudio.

\section{Complicaciones pos-CPRE}

La frecuencia en la presentación de complicaciones en este estudio fue baja ( $8 \%$ ). Ninguna de las complicaciones ocurridas fue estadísticamente significativa, de acuerdo con la edad. Las dos complicaciones más frecuentes en el estudio han sido descritas también como las más prevalentes (19, 21, 22-27) (tabla 5).

Tabla 5. Complicaciones pos-CPRE reportadas en otros estudios

\begin{tabular}{|c|c|c|c|c|}
\hline Ref. & $\begin{array}{l}\text { Rango } \\
\text { de edad }\end{array}$ & Diseño & n. ${ }^{\circ}$ & Complicaciones \\
\hline 23 & $65-94$ & Prospectivo & 50 & Ninguna \\
\hline 24 & $>65$ & Prospectivo & 677 & $\begin{array}{l}\text { Colangitis } 7(1,0 \%) \\
\text { Pancreatitis } 5(0,74 \%) \\
\text { Hemorragia } 3(0,44 \%) \\
\text { Perforación } 3(0,44 \%) \\
\text { Mortalidad } 2(0,29 \%)\end{array}$ \\
\hline 25 & $75-100$ & Retrospectivo & 101 & $\begin{array}{l}\text { Colangitis } 4(3,96 \%) \\
\text { Pancreatitis } 1(0,99 \%) \\
\text { Perforación } 1(0,99 \%)\end{array}$ \\
\hline 19 & $>70$ & Retrospectivo & 403 & $\begin{array}{l}\text { Pancreatitis } 9(2,23 \%) \\
\text { Hemorragia } 7(1,74 \%) \\
\text { Colangitis } 6(1,48 \%) \\
\text { Colecistitis } 3(0,74 \%) \\
\text { Insuficiencia hepática } 1(0,24 \%) \\
\text { Mortalidad } 1(0,24 \%)\end{array}$ \\
\hline 26 & $85-94$ & Prospectivo & 21 & Pancreatitis $1(4,76 \%)$ \\
\hline 27 & $>90$ & Retrospectivo & 23 & Hemorragia 3 (13\%) \\
\hline 21 & $>90$ & Retrospectivo & 126 & $\begin{array}{l}\text { Colangitis } 2(1,58 \%) \\
\text { Hemorragia } 2(1,58 \%) \\
\text { Mortalidad } 4(3,17 \%)\end{array}$ \\
\hline
\end{tabular}

El presente estudio sugiere que la edad mayor de 70 años no es un factor que incremente la mortalidad de los pacientes sometidos a la CPRE por coledocolitiasis. Sin embargo, las complicaciones cardiovasculares y la pancreatitis, como previamente fue reportado, son factores que incrementan la mortalidad relacionada con su realización.

\section{CONCLUSIÓN}

La CPRE es un procedimiento que muestra un buen perfil de seguridad. La edad mayor de 70 años no mostró un incremento en la mortalidad o las complicaciones. Es necesario realizar estudios futuros prospectivos y con análisis estadísticos más robustos que permitan explorar con mayor certeza la seguridad del procedimiento.

\section{Agradecimientos}

Agradecemos al Servicio de Cirugía General y Gastroenterología del Hospital Universitario Clínica San Rafael por apoyarnos durante la realización de este proyecto.

\section{REFERENCIAS}

1. Ross SO, Forsmark CE. Pancreatic and biliary disorders in the elderly. Gastroenterol Clin North Am. 2001;30:531-45.

2. Attasaranya S, Fogel EL, Lehman GA. Choledocholithiasis, ascending cholangitis, and gallstone pancreatitis. Med Clin North Am. 2008;92:925-60.

3. Freeman ML. Complications of endoscopic retrograde cholangiopancreatography: avoidance and management. Gastrointest Endosc Clin N Am. 2012;22:567-86.

4. Freeman ML, Nelson DB, Sherman S, Haber GB, Herman ME, Dorsher PJ, et al. Complications of endoscopic biliary sphincterotomy. N Engl J Med. 1996;335:909-18.

5. Ángel A, Rosero GVJ. Guía de manejo de coledocolitiasis. Bogotá: Hospital Universitario Clínica San Rafael; 2009. p. 6-7.

6. Peñaloza-Ramírez A, Leal-Buitrago C, Rodríguez-Hernández A. Adverse events of ERCP at San José Hospital of Bogotá (Colombia). Rev Esp Enferm Dig. 2009; 101:837-49.

7. Cotton PB, Lehman G, Vennes J, Geenen JE, Russell RC, Meyers WC, et al. Endoscopic sphincterotomy complications and their management: an attempt at consensus. Gastrointest Endosc. 1991;37:383-93.

8. Mayumi T, Someya K, Ootubo H, Takama T, Kido T, Kamezaki F, et al. Progression of Tokyo Guidelines and Japanese Guidelines for management of acute cholangitis and cholecystitis. J UOEH. 2013;35:249-57.

9. Frossard JL, Morel PM. Detection and management of bile duct stones. Gastrointest Endosc. 2010;72:808-16.

10. Balmadrid B, Kozarek R. Prevention and management of adverse events of endoscopic retrograde cholangiopancreatography. Gastrointest Endosc Clin N Am. 2013;23:385-403.

11. Li C, Ford ES, Zhao G, Wen X-J, Gotway CA. Age adjustment of diabetes prevalence: Use of 2010 U.S. Census data. J Diabetes. 2014;6:451-61.

12. Bao YS, Jia XB, Ji Y, Yang J, Zhao S-L, Na S-P. High prevalence and risk factors for kidney dysfunction in patients with atherosclerotic cardio-cerebrovascular disease. QJM. 2014;107:443-9.

13. Wolfgang CL. Predicting complicated choledocholithiasis. J Surg Res. 2013;185:502-3.

14. Yriberry Ureña S, Monge Zapa V. Alterations in laboratory tests as predictors of choledocholithiasis in patients who underwent ERCP: experience in a national private center. Rev Gastroenterol Peru. 2007;27:253-8. 
15. Damnjanović Z, Jovanović $M$, Nagorni A, Radojković $M$, Sokolović D, Damnjanović G, et al. Correlation of inflammation parameters and biochemical markers of cholestasis with the intensity of lipid peroxidation in patients with choledocholithiasis. Vojnosanit Pregl. 2013;70:170-6.

16. Pereira-Lima JC, Jakobs R, Winter UH, Benz C, Martin WR, Adamek HE, et al. Long-term results ( 7 to 10 years) of endoscopic papillotomy for choledocholithiasis. Multivariate analysis of prognostic factors for the recurrence of biliary symptoms. Gastrointest Endosc. 1998;48:457-64.

17. Zippi M, De Felici I, Pica R, Occhigrossi G, Traversa G. Comparison of endoscopic retrograde cholangiopancreatography between elderly and younger patients for common bile duct stones. Clin Ter. 2013;164:e353-8.

18. Tantau M, Mercea V, Crisan D, Tantau A, Mester G, Vesa S, et al. ERCP on a cohort of 2,986 patients with cholelitiasis: a 10-year experience of a single center. J Gastrointestin Liver Dis. 2013;22:141-7.

19. Sugiyama M, Atomi Y. Endoscopic sphincterotomy for bile duct stones in patients 90 years of age and older. Gastrointest Endosc. 2000;52:187-91.

20. Obana T, Fujita N, Noda Y, Kobayashi G, Ito K, Horaguchi $\mathrm{J}$, et al. Efficacy and safety of therapeutic ERCP for the elderly with choledocholithiasis: comparison with younger patients. Intern Med. 2010;49:1935-41.
21. Rodríguez-González FJ, Naranjo-Rodríguez A, MataTapia I, Chicano-Gallardo M, Puente-Gutierrez JJ, LópezVallejos P, et al. ERCP in patients 90 years of age and older. Gastrointest Endosc. 2003;58:220-5.

22. Talar-Wojnarowska R, Szulc G, Woźniak B, Pazurek M, Małecka-Panas E. Assessment of frequency and safety of endoscopic retrograde cholangiopancreatography in patients over 80 years of age. Pol Arch Med Wewn. 2009;119:136-40.

23. MacMahon M, Walsh TN, Brennan P, Osborne H, Courtney MG. Endoscopic retrograde cholangiopancreatography in the elderly: a single unit audit. Gerontology. 1993;39:28-32.

24. Deans GT, Sedman P, Martin DF, Royston CM, Leow CK, Thomas WE, et al. Are complications of endoscopic sphincterotomy age related? Gut 1997;41:545-8.

25. Ashton CE, McNabb WR, Wilkinson ML, Lewis RR. Endoscopic retrograde cholangiopancreatography in elderly patients. Age Ageing. 1998;27:683-8.

26. Clarke GA, Jacobson BC, Hammett RJ, Carr-Locke DL. The indications, utilization and safety of gastrointestinal endoscopy in an extremely elderly patient cohort. Endoscopy. 2001;33:580-4.

27. Mitchell RMS, O’Connor F, Dickey W. Endoscopic retrograde cholangiopancreatography is safe and effective in patients 90 years of age and older. J Clin Gastroenterol. 2003;36:72-4. 